\title{
TRANSDIAGNOSTIC APPROACH ON CBT AND SCHEMA THERAPY: \\ REVISITING AND CURING MEMORIES BY IMAGERY RESCRIPTING AND ITS IMPACT ON AFFECT REGULATION
}

Érica de Lana- delanna.uff@gmail.com - Prof. PhD - Department of Psychology
UFF (Universidade Federal Fluminense) - Rio de Janeiro, Brazil

Background: Intrusive, emotional memories are in the basis of some psychopathological conditions; even in non-diagnosticated cases, emotional memories can be painful and incapacitating ((FIG.1)). ImRs is a therapeutic technique that aims to reduce the distress associated with negative memories of early aversive experiences. It consists of prompting patients to rescript the autobiographical memory in line with their unmet needs ((FIG.2)). Objectives: This work aims to: 1) characterize Imagery Rescripting (ImRs) technique as a useful tool on transdiagnostic psychotherapeutic approach; 2) describe the neurobiological underlying mechanisms that confers to ImRs its possibility of curing negative autobiographical memories linked to emotional, traumatic episodes ((FIG.3)); and 3) point out the influence of ImRs in the emotional regulation ((FIG.4)). Materials and methods: Exploratory review of the literature.

2
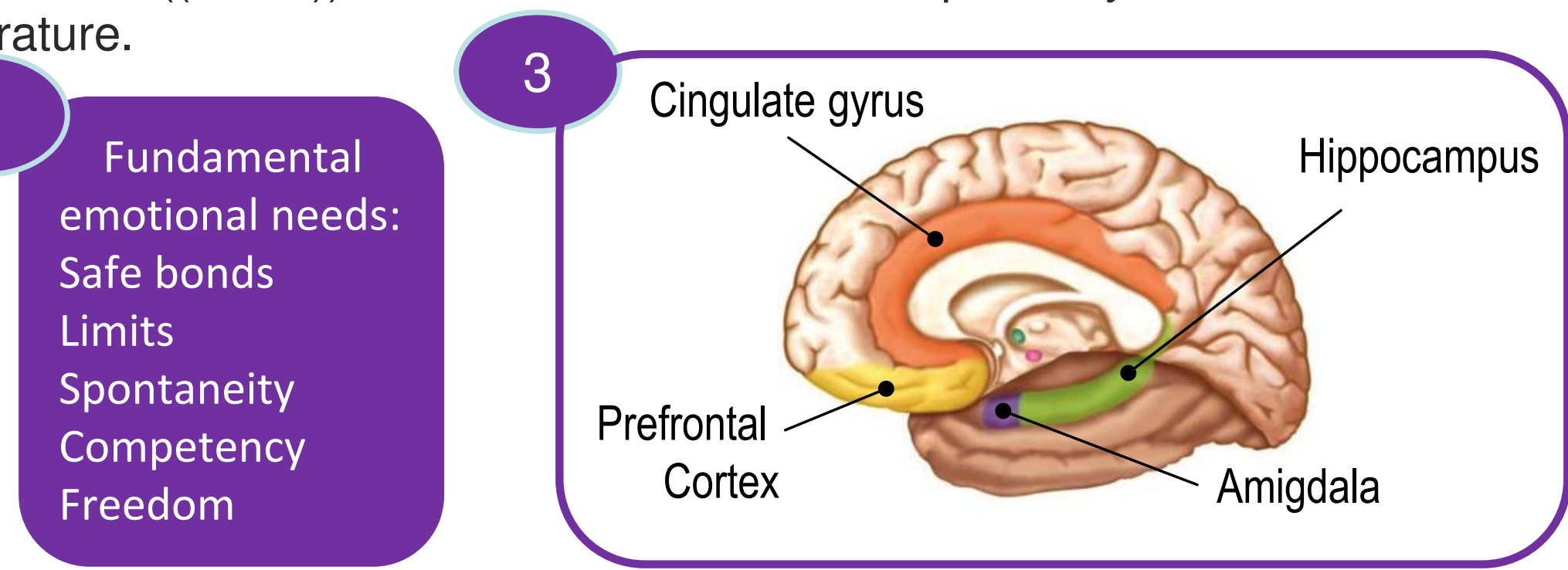

(Young, Klosko \& Weishaar, 2008)
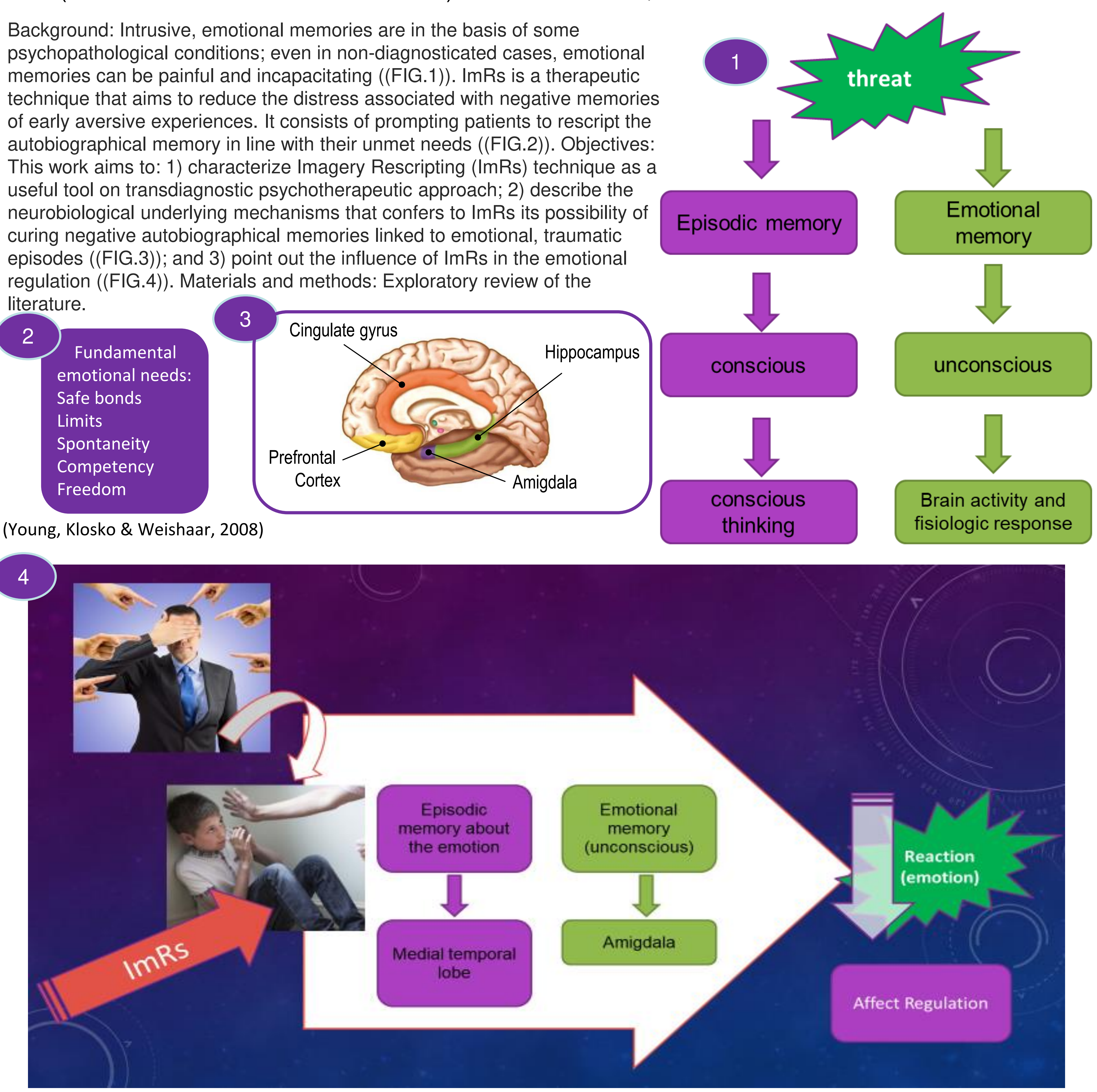

(de Lana, 2019)

Results and Conclusions: Empirical evidence has shown that ImRs reduces the negative self-belief derived from aversive memories in different types of mental disorders. ImRs changes the semantic self-representation encapsulated in the aversive memory by reducing the meta-emotional problem (i.e., perceiving a negative emotion as problematic and unacceptable). ImRs is a therapeutic technique addressing specific memories of earlier experiences associated with present problems. Neuroimaging studies indicate that remembering memories and imagining show marked overlap in brain activity. By imagining that the course of events is changed in a more desired direction, powerful therapeutic effects have been found, notably regarding emotional regulation. Alterations in some specific brain areas seem to be the neurobiological substrates in this reappraisal; such studies illustrate the potential for cross talk between cognitive neuroscience and clinical psychopathology research. 